\title{
Bounds on the multipartite entanglement of superpositions
}

\author{
Wei Song, ${ }^{1}$ Nai-Le Liu, ${ }^{1}$ and Zeng-Bing Chen ${ }^{1}$ \\ ${ }^{1}$ Hefei National Laboratory for Physical Sciences at Microscale and Department of Modern Physics, \\ University of Science and Technology of China, Hefei, Anhui 230026, China
}

\begin{abstract}
We derive the lower and upper bounds on the entanglement of a given multipartite superposition state in terms of the entanglement of the states being superposed. The first entanglement measure we use is the geometric measure, and the second is the q-squashed entanglement. These bounds allow us to estimate the amount of the multipartite entanglement of superpositions. We also show that two states of high fidelity to one another do not necessarily have nearly the same q-squashed entanglement.
\end{abstract}

PACS numbers: 03.67.-a, 03.67.Mn, 03.65.Ud

Recently, Linden et al. [1] raised the following problem: given a certain decomposition of a bipartite state $|\Gamma\rangle$ as a superposition of two other states, what is the relation between the entanglement of $|\Gamma\rangle$ and those of the two states being superposed? They derived upper bounds on the entanglement of $|\Gamma\rangle$ in terms of those of the two states in the superposition, where the entanglement was quantified by the von Neumann entropy. Subsequently, $\mathrm{Yu}$ et al. [2] and $\mathrm{Ou}$ et al. [3] investigated the same problem in terms of concurrence and negativity, respectively. Yet their results only apply to bipartite cases. The aim of this paper is to provide general lower and upper bounds on the entanglement of superposition states in multipartite scenarios. First we use the so-called geometric measure [4, 5]. This measure distinguishes itself in that it is suitable for any-partite systems with arbitrary dimensions. Then we use the q-squashed entanglement [6]. Among all existing entanglement measures, only the q-squashed entanglement has been proved to be additive both for bipartite states and for multipartite states [7].

We begin by briefly reviewing the definition of geometric measure. Given a general $k$-partite pure state $|\psi\rangle$, the geometric measure is defined as [5]

$$
E_{g}(|\psi\rangle)=1-\Lambda_{\max }^{k}(|\psi\rangle),
$$

where $\Lambda_{\max }^{k}(|\psi\rangle)=\sup _{|\phi\rangle \in S_{k}}|\langle\psi \mid \phi\rangle|^{2}$ with $S_{k}$ being the set of $k$-separable states. In terms of the geometric measure we have a lower bound on the entanglement of a multipartite superposition state, as formulated in the following theorem:

Theorem 1: Let $\left|\psi_{1}\right\rangle$ and $\left|\psi_{2}\right\rangle$ be arbitrary normalized $k$-partite pure states. The geometric measure of their superposed states $|\Gamma\rangle=\frac{a\left|\psi_{1}\right\rangle+b\left|\psi_{2}\right\rangle}{\| a\left|\psi_{1}\right\rangle+b\left|\psi_{2}\right\rangle \|}$ with $|a|^{2}+|b|^{2}=1$ satisfies

$$
\begin{aligned}
& \| a\left|\psi_{1}\right\rangle+b\left|\psi_{2}\right\rangle \|^{2} E_{g}(|\Gamma\rangle) \geq \max \left\{|a|^{2} E_{g}\left(\left|\psi_{1}\right\rangle\right)\right. \\
& +|b|^{2} E_{g}\left(\left|\psi_{2}\right\rangle\right)+2\left[\operatorname{Re}\left(a^{*} b\left\langle\psi_{1} \mid \psi_{2}\right\rangle\right)\right. \\
& \left.\left.-|a b| \sqrt{1-E_{g}\left(\left|\psi_{1}\right\rangle\right)} \sqrt{1-E_{g}\left(\left|\psi_{2}\right\rangle\right)}\right], 0\right\}
\end{aligned}
$$

Proof: Suppose $|\phi\rangle$ is the optimal $k$-separable state for
$|\Gamma\rangle$, i.e., the separable state closest to $|\Gamma\rangle$. Then we have

$$
\begin{aligned}
\Lambda_{\max }^{k}(|\Gamma\rangle) & =\frac{1}{\| a\left|\psi_{1}\right\rangle+b\left|\psi_{2}\right\rangle \|^{2}}\left\{|a|^{2}\left|\left\langle\psi_{1} \mid \phi\right\rangle\right|^{2}+|b|^{2}\left|\left\langle\psi_{2} \mid \phi\right\rangle\right|^{2}\right. \\
& \left.+2 \operatorname{Re}\left[a^{*} b\left\langle\psi_{1} \mid \phi\right\rangle\left\langle\phi \mid \psi_{2}\right\rangle\right]\right\} \\
& \leq \frac{1}{\| a\left|\psi_{1}\right\rangle+b\left|\psi_{2}\right\rangle \|^{2}}\left\{|a|^{2} \Lambda_{\max }^{k}\left(\left|\psi_{1}\right\rangle\right)\right. \\
& \left.+|b|^{2} \Lambda_{\max }^{k}\left(\left|\psi_{2}\right\rangle\right)+2|a b| \sqrt{\Lambda_{\max }^{k}\left(\left|\psi_{1}\right\rangle\right) \Lambda_{\max }^{k}\left(\left|\psi_{2}\right\rangle\right)}\right\} .
\end{aligned}
$$

By some simple algebraic calculation, we obtain

$$
\begin{aligned}
& \| a\left|\psi_{1}\right\rangle+b\left|\psi_{2}\right\rangle \|^{2} E_{g}(|\Gamma\rangle) \geq|a|^{2} E_{g}\left(\left|\psi_{1}\right\rangle\right) \\
& +|b|^{2} E_{g}\left(\left|\psi_{2}\right\rangle\right)+2\left[\operatorname{Re}\left(a^{*} b\left\langle\psi_{1} \mid \psi_{2}\right\rangle\right)\right. \\
& \left.-|a b| \sqrt{1-E_{g}\left(\left|\psi_{1}\right\rangle\right)} \sqrt{1-E_{g}\left(\left|\psi_{2}\right\rangle\right)}\right]
\end{aligned}
$$

where the lower bound is saturated if $\left|\psi_{1}\right\rangle=\left|\psi_{2}\right\rangle$. Because the geometric measure must be a nonnegative value, the proof is completed.

Below we will derive an upper bound for the geometric measure of the superposition state $|\Gamma\rangle$. For this purpose we use a lower bound less stringent than that in Eq. (4). As a consequence of Eq. (3) we have

$$
\begin{aligned}
\Lambda_{\max }^{k}(|\Gamma\rangle) & \leq \frac{1}{\| a\left|\psi_{1}\right\rangle+b\left|\psi_{2}\right\rangle \|^{2}}\left\{|a|^{2} \Lambda_{\max }^{k}\left(\left|\psi_{1}\right\rangle\right)\right. \\
& \left.+|b|^{2} \Lambda_{\max }^{k}\left(\left|\psi_{2}\right\rangle\right)+|a b|\left[\Lambda_{\max }^{k}\left(\left|\psi_{1}\right\rangle\right)+\Lambda_{\max }^{k}\left(\left|\psi_{2}\right\rangle\right)\right]\right\} .
\end{aligned}
$$

From this inequality it follows immediately that

$$
\begin{aligned}
& \| a\left|\psi_{1}\right\rangle+b\left|\psi_{2}\right\rangle \|^{2} E_{g}(|\Gamma\rangle) \\
\geq & |a(a+b)| E_{g}\left(\left|\psi_{1}\right\rangle\right)+|b(a+b)| E_{g}\left(\left|\psi_{2}\right\rangle\right) \\
& +2\left[\operatorname{Re}\left(a^{*} b\left\langle\psi_{1} \mid \psi_{2}\right\rangle\right)-|a b|\right] .
\end{aligned}
$$

Having the lower bound above, we can prove the following result.

Theorem 2. Let $\left|\psi_{1}\right\rangle$ and $\left|\psi_{2}\right\rangle$ be arbitrary normalized $k$-partite pure states. The geometric measure of their superposed states $|\Gamma\rangle=\frac{a\left|\psi_{1}\right\rangle+b\left|\psi_{2}\right\rangle}{\| a\left|\psi_{1}\right\rangle+b\left|\psi_{2}\right\rangle \|}$ with $|a|^{2}+|b|^{2}=1$ 
satisfies

$$
\| a\left|\psi_{1}\right\rangle+b\left|\psi_{2}\right\rangle \|^{2} E_{g}(|\Gamma\rangle) \leq \min \left\{A, B, \| a\left|\psi_{1}\right\rangle+b\left|\psi_{2}\right\rangle \|^{2}\right\},
$$

where

$$
\begin{aligned}
A= & \frac{1}{\left.|||a| \psi_{1}\right\rangle+b\left|\psi_{2}\right\rangle||-b \mid}\left\{|a|^{2} E_{g}\left(\left|\psi_{1}\right\rangle\right)\right. \\
& \left.-|b||||a| \psi_{1}\right\rangle+b\left|\psi_{2}\right\rangle \|-b \mid E_{g}\left(\left|\psi_{2}\right\rangle\right) \\
& +2\left[\operatorname{Re}\left(a^{*} b\left\langle\psi_{1} \mid \psi_{2}\right\rangle+|b|^{2}\right)\right. \\
& \left.\left.+|b| \| a\left|\psi_{1}\right\rangle+b\left|\psi_{2}\right\rangle||\right]\right\}
\end{aligned}
$$

and

$$
\begin{aligned}
B= & \frac{1}{\left.|\| a| \psi_{1}\right\rangle+b\left|\psi_{2}\right\rangle|\|-a|}\left\{|b|^{2} E_{g}\left(\left|\psi_{2}\right\rangle\right)\right. \\
& \left.-|a||||a| \psi_{1}\right\rangle+b\left|\psi_{2}\right\rangle \|-a \mid E_{g}\left(\left|\psi_{1}\right\rangle\right) \\
& +2\left[\operatorname{Re}\left(a b^{*}\left\langle\psi_{2} \mid \psi_{1}\right\rangle+|a|^{2}\right)\right. \\
& \left.\left.+|a| \| a\left|\psi_{1}\right\rangle+b\left|\psi_{2}\right\rangle \|\right]\right\} .
\end{aligned}
$$

Proof: To prove theorem 2, it is convenient to rewrite $\left|\psi_{1}\right\rangle$ as

$$
\left|\psi_{1}\right\rangle=\frac{\frac{\| a\left|\psi_{1}\right\rangle+b\left|\psi_{2}\right\rangle \|}{\sqrt{\| a\left|\psi_{1}\right\rangle+b\left|\psi_{2}\right\rangle \|^{2}+|b|^{2}}}}{\frac{a}{\sqrt{\| a\left|\psi_{1}\right\rangle+b\left|\psi_{2}\right\rangle \|^{2}+|b|^{2}}}}|\Gamma\rangle-\frac{\frac{b}{\sqrt{\| a\left|\psi_{1}\right\rangle+b\left|\psi_{2}\right\rangle \|^{2}+|b|^{2}}}}{\frac{a}{\sqrt{\| a\left|\psi_{1}\right\rangle+b\left|\psi_{2}\right\rangle \|^{2}+|b|^{2}}}}\left|\psi_{2}\right\rangle .
$$

Applying Eq. (6) to $\left|\psi_{1}\right\rangle$, we get

$$
\begin{aligned}
|a|^{2} E_{g}\left(\left|\psi_{1}\right\rangle\right) \geq & \| a\left|\psi_{1}\right\rangle+b\left|\psi_{2}\right\rangle \| \\
& \left.\times|\| a| \psi_{1}\right\rangle+b\left|\psi_{2}\right\rangle \|-b \mid E_{g}(|\Gamma\rangle) \\
& \left.+|b||||a| \psi_{1}\right\rangle+b\left|\psi_{2}\right\rangle \|-b \mid E_{g}\left(\left|\psi_{2}\right\rangle\right) \\
& -2\left[\operatorname{Re}\left(a^{*} b\left\langle\psi_{1} \mid \psi_{2}\right\rangle+|b|^{2}\right)\right. \\
& \left.+|b| \| a\left|\psi_{1}\right\rangle+b\left|\psi_{2}\right\rangle \|\right]
\end{aligned}
$$

from which it follows that

$$
\| a\left|\psi_{1}\right\rangle+b\left|\psi_{2}\right\rangle \| E_{g}(|\Gamma\rangle) \leq A
$$

where the upper bound $A$ is given in Eq. (8). The upper bound $B$ in Eq. (9) is obtained from $A$ by simply exchanging $a\left|\psi_{1}\right\rangle$ and $b\left|\psi_{2}\right\rangle$. The upper bound $\| a\left|\psi_{1}\right\rangle+b\left|\psi_{2}\right\rangle \|^{2}$ in Eq. (77) is due to the fact that the geometric measure is less than or equal to 1 .

Example 1: Consider the following superposed state

$$
|\Gamma\rangle=a|\mathrm{GHZ}\rangle+b|W\rangle
$$

where $|\mathrm{GHZ}\rangle=(1 / \sqrt{2})(|000\rangle+|111\rangle)$ and $|W\rangle=$ $(1 / \sqrt{3})(|001\rangle+|010\rangle+|100\rangle)$. Without loss of generality, we assume that $a$ and $b$ are both positive real numbers with $a^{2}+b^{2}=1$. The geometric measures of $|\mathrm{GHZ}\rangle$ and $|W\rangle$ have been computed in Ref. [8] to be $E_{g}(|\mathrm{GHZ}\rangle)=1 / 2$ and $E_{g}(|W\rangle)=5 / 9$. Inserting these results into Eq. (2) and Eq. (7) yields

$$
\begin{aligned}
E_{g}(|\Gamma\rangle) \geq & \max \left\{-\frac{1}{18} a^{2}-\frac{4}{3 \sqrt{2}} a \sqrt{1-a^{2}}+\frac{5}{9}, 0\right\} \\
E_{g}(|\Gamma\rangle) \leq & \min \left\{\frac{1}{1-a}\left(\frac{35}{18} a^{2}+\frac{3}{2} a+\frac{5}{9}\right),\right. \\
& \left.\frac{1}{1-\sqrt{1-a^{2}}}\left(-\frac{37}{18} a^{2}+\frac{13}{9} \sqrt{1-a^{2}}+\frac{23}{9}\right), 1\right\} .
\end{aligned}
$$

The lower and upper bounds vs $a$ are shown in Fig.1.

For a superposition of more than two components we can prove the following proposition by the same way as proving Theorem 1.

Proposition: For a superposed state $|\Gamma\rangle=$ $\frac{a_{1}\left|\psi_{1}\right\rangle+\cdots+a_{n}\left|\psi_{n}\right\rangle}{\| a_{1}\left|\psi_{1}\right\rangle+\cdots+a_{n}\left|\psi_{n}\right\rangle \|}$ with $\sum_{i=1}^{n}\left|a_{i}\right|^{2}=1$, the following inequality holds

$$
\begin{aligned}
& \| a_{1}\left|\psi_{1}\right\rangle+\cdots+a_{n}\left|\psi_{n}\right\rangle \|^{2} E_{g}(|\Gamma\rangle) \\
& \geq \max \left\{\left|a_{1}\right|^{2} E_{g}\left(\left|\psi_{1}\right\rangle\right)+\cdots+\left|a_{n}\right|^{2} E_{g}\left(\left|\psi_{n}\right\rangle\right)+\sum_{k, l=1, k \neq l}^{n}\left[a_{k}^{*} a_{l}\left\langle\psi_{k} \mid \psi_{l}\right\rangle-\left|a_{k} a_{l}\right| \sqrt{1-E_{g}\left(\left|\psi_{k}\right\rangle\right)} \sqrt{1-E_{g}\left(\left|\psi_{l}\right\rangle\right)}\right], 0\right\}
\end{aligned}
$$

In the remainder of this paper, we shall derive an upper bound and a lower bound in terms of the multipartite q-squashed entanglement [6]. For an $N$-partite state $\rho_{A_{1}, \ldots, A_{N}}$, the q-squashed entanglement is defined as

$$
E_{\mathrm{sq}}^{\mathrm{q}}\left(\rho_{A_{1}, \ldots, A_{N}}\right)=\inf I\left(A_{1}: A_{2}: \cdots: A_{N} \mid E\right),
$$

where the infimum is taken over all states $\sigma_{A_{1}, \ldots, A_{N}, E}$, that are extensions of $\rho_{A_{1}, \ldots, A_{N}}$, i.e., $\operatorname{Tr}_{E} \sigma=\rho$. For an
$N$-partite pure state $|\Gamma\rangle_{A_{1}, \ldots, A_{N}}$, we have

$$
E_{\mathrm{sq}}^{\mathrm{q}}\left(|\Gamma\rangle_{A_{1}, \ldots, A_{N}}\right)=S\left(\rho_{A_{1}}\right)+\cdots+S\left(\rho_{A_{N}}\right),
$$

where $\rho_{A_{k}}$ is obtained from $|\Gamma\rangle\langle\Gamma|$ by tracing out the $k$-th component. We can prove the following theorem:

Theorem 3 Let $\left|\psi_{1}\right\rangle$ and $\left|\psi_{2}\right\rangle$ be arbitrary normalized $N$-partite pure states. The q-squashed entanglement of 


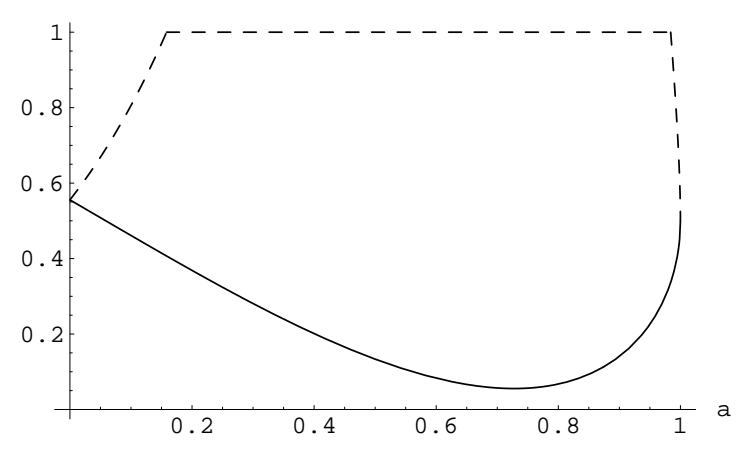

FIG. 1: The lower and upper bounds of geometric measure of $|\Gamma\rangle$ vs $a$. The dash line corresponds to the upper bound, and the solid line corresponds to the lower bound.

their superposed state $|\Gamma\rangle=\frac{a\left|\psi_{1}\right\rangle+b\left|\psi_{2}\right\rangle}{\| a\left|\psi_{1}\right\rangle+b\left|\psi_{2}\right\rangle \|}$ with $|a|^{2}+$ $|b|^{2}=1$ satisfies

$$
\begin{aligned}
& \| a\left|\psi_{1}\right\rangle+b\left|\psi_{2}\right\rangle \|^{2} E_{\mathrm{sq}}^{\mathrm{q}}(|\Gamma\rangle) \\
\leq & 2\left[|a|^{2} E_{\mathrm{sq}}^{\mathrm{q}}\left(\left|\psi_{1}\right\rangle\right)+|b|^{2} E_{\mathrm{sq}}^{\mathrm{q}}\left(\left|\psi_{2}\right\rangle\right)+N h_{2}\left(|a|^{2}\right)\right] .
\end{aligned}
$$

Proof: To prove this, let us consider the state

$\rho_{A_{1}}^{\prime}=|a|^{2} \operatorname{Tr}_{A_{2}, \ldots, A_{N}}\left(\left|\psi_{1}\right\rangle\left\langle\psi_{1}\right|\right)+|b|^{2} \operatorname{Tr}_{A_{2}, \ldots, A_{N}}\left(\left|\psi_{2}\right\rangle\left\langle\psi_{2}\right|\right)$,

Recalling the property $S\left(|a|^{2} \rho+|b|^{2} \sigma\right) \leq|a|^{2} S(\rho)+$ $|b|^{2} S(\sigma)+h_{2}\left(|a|^{2}\right)$, where $h_{2}(x)=-x \log _{2} x-(1-$ $x) \log _{2}(1-x)$ is the binary entropy function, we have

$$
\begin{aligned}
S\left(\rho_{A_{1}}^{\prime}\right) \leq & |a|^{2} S\left(\operatorname{Tr}_{A_{2}, \ldots, A_{N}}\left|\psi_{1}\right\rangle\left\langle\psi_{1}\right|\right) \\
& +|b|^{2} S\left(\operatorname{Tr}_{A_{2}, \ldots, A_{N}}\left|\psi_{2}\right\rangle\left\langle\psi_{2}\right|\right)+h_{2}\left(|a|^{2}\right) .
\end{aligned}
$$

On the other hand, $\rho_{A_{1}}^{\prime}$ can also be written as

$$
\begin{aligned}
& \rho_{A_{1}}^{\prime}=\frac{\| a\left|\psi_{1}\right\rangle+b\left|\psi_{2}\right\rangle \|^{2}}{2} \\
& \times \operatorname{Tr}_{A_{2}, \ldots, A_{N}}\left[\frac{\left(a\left|\psi_{1}\right\rangle+b\left|\psi_{2}\right\rangle\right)}{\| a\left|\psi_{1}\right\rangle+b\left|\psi_{2}\right\rangle \|} \frac{\left(a^{*}\left\langle\psi_{1}\right|+b^{*}\left\langle\psi_{2}\right|\right)}{\| a\left|\psi_{1}\right\rangle+b\left|\psi_{2}\right\rangle \|}\right] \\
& +\frac{\| a\left|\psi_{1}\right\rangle-b\left|\psi_{2}\right\rangle \|^{2}}{2} \\
& \times \operatorname{Tr}_{A_{2}, \ldots, A_{N}}\left[\frac{\left(a\left|\psi_{1}\right\rangle-b\left|\psi_{2}\right\rangle\right)}{\| a\left|\psi_{1}\right\rangle-b\left|\psi_{2}\right\rangle \|} \frac{\left(a^{*}\left\langle\psi_{1}\right|-b^{*}\left\langle\psi_{2}\right|\right)}{\| a\left|\psi_{1}\right\rangle-b\left|\psi_{2}\right\rangle \|}\right] .
\end{aligned}
$$

From the concavity of von Neumann entropy one has

$$
\begin{aligned}
& \frac{\| a\left|\psi_{1}\right\rangle+b\left|\psi_{2}\right\rangle \|^{2}}{2} S\left(\operatorname{Tr}_{A_{2}, \ldots, A_{N}}|\Gamma\rangle\langle\Gamma|\right) \\
& +\frac{\| a\left|\psi_{1}\right\rangle-b\left|\psi_{2}\right\rangle \|^{2}}{2} S\left(\operatorname{Tr}_{A_{2}, \ldots, A_{N}}|\bar{\Gamma}\rangle\langle\bar{\Gamma}|\right) \leq S\left(\rho_{A_{1}}^{\prime}\right),
\end{aligned}
$$

where $|\bar{\Gamma}\rangle=\frac{a\left|\psi_{1}\right\rangle-b\left|\psi_{2}\right\rangle}{\| a\left|\psi_{1}\right\rangle-b\left|\psi_{2}\right\rangle \|}$. Combining Eq. (19) and Eq. (21) leads to

$$
\begin{aligned}
& \| a\left|\psi_{1}\right\rangle+b\left|\psi_{2}\right\rangle \|^{2} S\left(\operatorname{Tr}_{A_{2}, \ldots, A_{N}}|\Gamma\rangle\langle\Gamma|\right) \\
& +\| a\left|\psi_{1}\right\rangle-b\left|\psi_{2}\right\rangle \|^{2} S\left(\operatorname{Tr}_{A_{2}, \ldots, A_{N}}|\bar{\Gamma}\rangle\langle\bar{\Gamma}|\right) \\
\leq & 2\left[|a|^{2} S\left(\operatorname{Tr}_{A_{2}, \ldots, A_{N}}\left|\psi_{1}\right\rangle\left\langle\psi_{1}\right|\right)\right. \\
& \left.+|b|^{2} S\left(\operatorname{Tr}_{A_{2}, \ldots, A_{N}}\left|\psi_{2}\right\rangle\left\langle\psi_{2}\right|\right)+h_{2}\left(|a|^{2}\right)\right] .
\end{aligned}
$$

Since $S\left(\operatorname{Tr}_{A_{2}, \ldots, A_{N}}|\bar{\Gamma}\rangle\langle\bar{\Gamma}|\right) \geq 0$, it follows that

$$
\begin{aligned}
& \| a\left|\psi_{1}\right\rangle+b\left|\psi_{2}\right\rangle \|^{2} S\left(\operatorname{Tr}_{A_{2}, \ldots, A_{N}}|\Gamma\rangle\langle\Gamma|\right) \\
& \leq 2\left[|a|^{2} S\left(\operatorname{Tr}_{A_{2}, \ldots, A_{N}}\left|\psi_{1}\right\rangle\left\langle\psi_{1}\right|\right)\right. \\
& \left.+|b|^{2} S\left(\operatorname{Tr}_{A_{2}, \ldots, A_{N}}\left|\psi_{2}\right\rangle\left\langle\psi_{2}\right|\right)+h_{2}\left(|a|^{2}\right)\right] .
\end{aligned}
$$

Similarly, we can deduce the following inequalities

$$
\begin{aligned}
& \| a\left|\psi_{1}\right\rangle+b\left|\psi_{2}\right\rangle \|^{2} S\left(\operatorname{Tr}_{A_{1}, \ldots, A_{k-1} A_{k+1}, \ldots, A_{N}}|\Gamma\rangle\langle\Gamma|\right) \\
\leq & 2\left[|a|^{2} S\left(\operatorname{Tr}_{A_{1}, \ldots, A_{k-1} A_{k+1}, \ldots, A_{N}}\left|\psi_{1}\right\rangle\left\langle\psi_{1}\right|\right)\right. \\
& \left.+|b|^{2} S\left(\operatorname{Tr}_{A_{1}, \ldots, A_{k-1} A_{k+1}, \ldots, A_{N}}\left|\psi_{2}\right\rangle\left\langle\psi_{2}\right|\right)+h_{2}\left(|a|^{2}\right)\right]
\end{aligned}
$$

for $k=1, \ldots, N$. Adding all these inequalities together and using Eq. (16), the advertised inequality in Eq. (17) is proved.

Example 2: Consider the following $N$-partite states:

$$
\begin{aligned}
\left|\psi_{1}\right\rangle & =\sqrt{\frac{1}{10}}|1\rangle^{\otimes N}+\sqrt{\frac{9}{10}} \sqrt{\frac{1}{d-1}}\left(|2\rangle^{\otimes N}+\cdots+|d\rangle^{\otimes N}\right), \\
\left|\psi_{2}\right\rangle & =\sqrt{\frac{1}{10}}|1\rangle^{\otimes N}-\sqrt{\frac{9}{10}} \sqrt{\frac{1}{d-1}}\left(|2\rangle^{\otimes N}+\cdots+|d\rangle^{\otimes N}\right), \\
a & =-b=\frac{1}{\sqrt{2}} .
\end{aligned}
$$

We fix $d=11$ and consider $N \leq 8$. For each superposed state we calculate $E_{\mathrm{sq}}^{\mathrm{q}}(|\Gamma\rangle)$ and its upper bound. The results are shown in Fig.2(a). On the other hand, for $N=$ 3 and $d \leq 8$, the values of $E_{\mathrm{sq}}^{\mathrm{q}}(|\Gamma\rangle)$ and the corresponding upper bounds are shown in Fig.2(b). One sees that each q-squashed entanglement diverges from its upper bound not too much in all these cases.

Recently, Gour [9] derived tight lower and upper bounds on the entanglement (von Neumann entropy) of a superposition of two bipartite states in terms of the entanglement of the two states constituting the superposition. The new upper bound is tighter than the one presented in [1]. Gour's upper bound leads immediately to a new upper bound for the q-squashed entanglement of an $N$-partite pure state; the new upper bound is more stringent than the one given in Theorem 3.

Theorem 4. Let $\left|\psi_{1}\right\rangle$ and $\left|\psi_{2}\right\rangle$ be arbitrary normalized $N$-partite pure states. The q-squashed entanglement of their superposed states $|\Gamma\rangle=\frac{a\left|\psi_{1}\right\rangle+b\left|\psi_{2}\right\rangle}{\| a\left|\psi_{1}\right\rangle+b\left|\psi_{2}\right\rangle \|}$ with $|a|^{2}+$ $|b|^{2}=1$ satisfies

$$
\| a\left|\psi_{1}\right\rangle+b\left|\psi_{2}\right\rangle \|^{2} E_{\mathrm{sq}}^{\mathrm{q}}(|\Gamma\rangle) \leq f(t)
$$



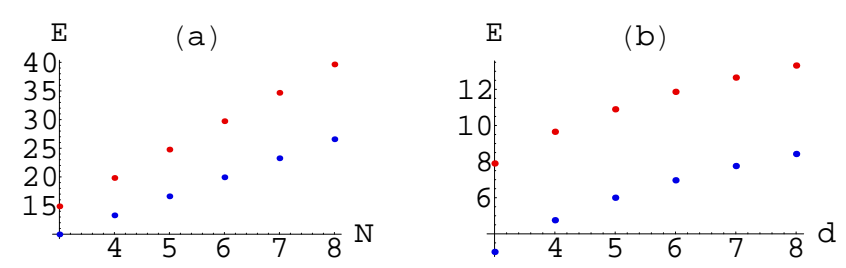

FIG. 2: Color online. (a)Plots of the q-squashed entanglement of superposed states (blue dots) and upper bounds (red dots) for $d=11$ and $N \leq 8,(\mathrm{~b}) N=3$ and $d \leq 8$.

for all $0 \leq t \leq 1$, where

$$
\begin{aligned}
f(t)= & \frac{t|b|^{2}+(1-t)|a|^{2}}{t(1-t)}\left[t E_{\mathrm{sq}}^{\mathrm{q}}\left(\left|\psi_{1}\right\rangle\right)\right. \\
& \left.+(1-t) E_{\mathrm{sq}}^{\mathrm{q}}\left(\left|\psi_{2}\right\rangle\right)+N h_{2}(t)\right] .
\end{aligned}
$$

Here, the minimum of the function $f(t)$ is achieved when $t$ satisfies the equation

$$
\frac{|a|^{2}(1-t)^{2}}{|b|^{2} t^{2}}=\frac{E_{\mathrm{sq}}^{\mathrm{q}}\left(\left|\psi_{1}\right\rangle\right)-N \log _{2} t}{E_{\mathrm{sq}}^{\mathrm{q}}\left(\left|\psi_{2}\right\rangle\right)-N \log _{2}(1-t)}
$$

Gour's lower bound, on the other hand, enables us to obtain a lower bound for the q-squashed entanglement of an $N$-partite pure state:

Theorem 5. Let $\left|\psi_{1}\right\rangle$ and $\left|\psi_{2}\right\rangle$ be arbitrary $N$-partite pure states, and let $|\Gamma\rangle=a\left|\psi_{1}\right\rangle+b\left|\psi_{2}\right\rangle$ be a normalized state. Then the q-squashed entanglement of $|\Gamma\rangle$ satisfies

$$
E_{\mathrm{sq}}^{\mathrm{q}}(|\Gamma\rangle) \geq \max \{C(t), D(t)\} .
$$

for all $0 \leq t \leq 1$, where

$$
\begin{aligned}
& C(t)=\frac{(1-t)|b|^{2}}{1-t\left(1-|a|^{2}\right)} E_{\mathrm{sq}}^{\mathrm{q}}\left(\left|\psi_{2}\right\rangle\right)-\frac{1-t}{t} E_{\mathrm{sq}}^{\mathrm{q}}\left(\left|\psi_{1}\right\rangle\right)-\frac{N}{t} h_{2}(t), \\
& D(t)=\frac{(1-t)|a|^{2}}{1-t\left(1-|b|^{2}\right)} E_{\mathrm{sq}}^{\mathrm{q}}\left(\left|\psi_{1}\right\rangle\right)-\frac{1-t}{t} E_{\mathrm{sq}}^{\mathrm{q}}\left(\left|\psi_{2}\right\rangle\right)-\frac{N}{t} h_{2}(t) .
\end{aligned}
$$

The maximum of $C(t)$ is obtained when

$$
\frac{|a|^{2}|b|^{2} t^{2}}{\left[1-\left(1-|a|^{2}\right) t\right]^{2}} E_{\mathrm{sq}}^{\mathrm{q}}\left(\left|\psi_{2}\right\rangle\right)=E_{\mathrm{sq}}^{\mathrm{q}}\left(\left|\psi_{1}\right\rangle\right)-N \log _{2}(1-t) .
$$

The analogous formula applies to $D(t)$.

Analogous to Ref. [1], we can show that if the entanglement is quantified by the multipartite q-squashed entanglement, then two states of high fidelity to one another do not necessarily have nearly the same entanglement.

Example 3: Suppose $\left|\psi_{1}\right\rangle=|000\rangle$, and $\left|\psi_{2}\right\rangle=$ $\sqrt{1-\varepsilon}\left|\psi_{1}\right\rangle+\sqrt{\varepsilon / d}(|111\rangle+\cdots+|d d d\rangle)$. It is easy to show that $E_{\mathrm{sq}}^{\mathrm{q}}\left(\left|\psi_{1}\right\rangle\right)=0$ and $E_{\mathrm{sq}}^{\mathrm{q}}\left(\left|\psi_{2}\right\rangle\right)=$ $3\left[-(1-\varepsilon) \log _{2}(1-\varepsilon)-d\left(\frac{\varepsilon}{d} \log _{2} \frac{\varepsilon}{d}\right)\right] \approx 3 \varepsilon \log _{2} d$. The fidelity $\left|\left\langle\psi_{1} \mid \psi_{2}\right\rangle\right|^{2}=1-\varepsilon$ approaches one for small $\varepsilon$, while the difference in the q-squashed entanglement of $\left|\psi_{1}\right\rangle$ and $\left|\psi_{2}\right\rangle$ can be as large as we expect if we choose an appropriate $d$.

Summarizing, we have presented lower and upper bounds on the entanglement of the multipartite superposition state in terms of the geometric measure and qsquashed entanglement measure, respectively. Our results partly solve the open problem proposed in Ref. [1]. In view of the fact that the geometric measure and the q-squashed entanglement measure are both multipartite entanglement measure, our results may find useful applications in future manipulations of multipartite entanglement.

We thank D. Yang for valuable suggestions and K. Chen for bringing Ref. [10] and [1] to our attention. This work is supported by the NNSF of China, the CAS, the National Fundamental Research Program (Grant No. 2006CB921900), and Anhui Provincial Natural Science Foundation (Grant No. 070412050).

Note added. After completing this manuscript, We became aware of two recently related papers by J. Niset et al. [10] and D. Cavalcanti et al. [11].
[1] N. Linden, S. Popescu, and J. A. Smolin, Phys. Rev. Lett. 97, 100502 (2006).

[2] C. S. Yu, X. X. Yi, and H. S. Song, Phys. Rev. A 75, $022332(2007)$.

[3] Y. C. Ou and H. Fan, Phys. Rev. A 76, 022320 (2007), e-print arXiv:quant-ph/0704.0757.

[4] A. Shimony, Ann. N.Y. Acad. Sci. 755, 675 (1995).

[5] H. Barnum and N. Linden, J. Phys. A: Math. Gen. 34, 6787 (2001).

[6] M. Christandl and A. Winter, J. Math. Phys. 45, 829
(2003).

[7] D. Yang, K. Horodecki, M. Horodecki, P. Horodecki, J. Oppenheim, W. Song, e-print arXiv:quant-ph/0704.2236.

[8] T.-C. Wei and P.M. Goldbart, Phys. Rev. A 68,042307 (2003).

[9] G. Gour, e-print arXiv:quant-ph/0707.1521.

[10] J. Niset, N. J. Cerf, e-print arXiv:quant-ph/0705.4650.

[11] D. Cavalcanti, M. O. Terra Cunha, A. Acin, e-print arXiv:quant-ph/0705.2521. 